\title{
A Robust Control Method for Q-S Synchronization between Different Dimensional Integer-Order and Fractional-Order Chaotic Systems
}

\author{
Adel Ouannas ${ }^{1}$ and Raghib Abu-Saris ${ }^{2}$ \\ ${ }^{1}$ Department of Mathematics and Computer Science, University of Tebessa, 12002 Tebessa, Algeria \\ ${ }^{2}$ Department of Health Informatics, College of Public Health and Health Informatics, King Saud Bin Abdulaziz University for \\ Health Science, Riyadh 11481, Saudi Arabia
}

Correspondence should be addressed to Adel Ouannas; ouannas_adel@yahoo.fr

Received 8 November 2015; Accepted 17 December 2015

Academic Editor: Xiao He

Copyright (C) 2015 A. Ouannas and R. Abu-Saris. This is an open access article distributed under the Creative Commons Attribution License, which permits unrestricted use, distribution, and reproduction in any medium, provided the original work is properly cited.

\begin{abstract}
A robust control approach is presented to study the problem of Q-S synchronization between Integer-order and fractionalorder chaotic systems with different dimensions. Based on Laplace transformation and stability theory of linear integer-order dynamical systems, a new control law is proposed to guarantee the Q-S synchronization between $n$-dimensional integer-order master system and $m$-dimensional fractional-order slave system. This paper provides further contribution to the topic of $Q-S$ chaos synchronization between integer-order and fractional-order systems and introduces a general control scheme that can be applied to wide classes of chaotic and hyperchaotic systems. Illustrative example and numerical simulations are used to show the effectiveness of the proposed method.
\end{abstract}

\section{Introduction}

Fractional derivatives provide an excellent instrument for the description of memory and hereditary properties of various materials and processes. The advantages or the real objects of the fractional-order systems are that we have more degrees of freedom in the model and that a "memory" is included in the model [1-6].

Recently, more and more attentions were paid to synchronization of integer-order chaotic systems and fractionalorder chaotic systems. Many control methods have been proposed and different synchronization types have been studied between integer-order and fractional-order chaotic system. For example, general control schemes have been described in $[7,8]$. A sliding mode method has been designed in [9-11]. A synchronization method of a class of hyperchaotic systems is given in [12]. In [13], a nonlinear feedback control method has been introduced and some robust observer techniques have been used in $[14,15]$. Also, complete synchronization and antisynchronization have been observed, for example, in [16-18], and function projective synchronization has been studied in [19]. Until now, a variety of control schemes have been proposed to study the problem of chaos synchronization between different dimensional systems such as modified function projective synchronization [20], generalized matrix projective synchronization [21], generalized synchronization [22-24], inverse generalized synchronization [25], full state hybrid projective synchronization [26], Q-S synchronization [27], increased order synchronization [28, 29], and reduced order generalized synchronization [30]. Amongst all kinds of synchronization, $Q-S$ synchronization has been extensively considered [31-39], due to its universality and its great potential applications in applied sciences and engineering.

Motivated by the above discussions, the main aim of this paper is to present constructive scheme to investigate $Q-S$ synchronization between $n$-dimensional integer-order master system and $m$-dimensional fractional-order slave system in $n$-D. By using Laplace transformation, stability property of integer-order linear systems, and suitable fractional-order control law, a new criterion is derived to achieve the Q-S 
chaos synchronization between $n$-dimensional integer-order master system and $m$-dimensional fractional-order slave system in $n$-D. The proposed control method is simple, efficient, and easy to implement in applications. The outline of the rest of this paper is organized as follows. First, Section 2 provides some basic concepts of fractional derivative. In Section 3, the problem of Q-S synchronization between integer-order master system and fractional-order slave system is formulated. Our main result is presented in Section 4. In Section 5, numerical example is used to verify the effectiveness and feasibility of the proposed control scheme. Finally, Section 6 is the brief conclusion.

\section{Basic Concepts of Fractional Derivative}

There are several definitions of a fractional derivative of order $\alpha>0$ [40-42]. The two most commonly used definitions are the Riemann-Liouville and Caputo definitions. Each definition uses Riemann-Liouville fractional integration and derivatives of whole order. The difference between the two definitions is in the order of evaluation. The RiemannLiouville fractional integral operator of order $\alpha \geq 0$ of the function $f(t)$ is defined as

$$
J^{\alpha} f(t)=\frac{1}{\Gamma(\alpha)} \int_{0}^{t}(t-\tau)^{\alpha-1} f(\tau) d \tau, \quad \alpha>0, t>0 .
$$

Some properties of the operator $J^{\alpha}$ can be found, for example, in $[43,44]$. We recall only the following, for $\mu \geq-1, \alpha, \beta \geq 0$, and $\gamma>-1$; we have

$$
\begin{aligned}
J^{\alpha} J^{\beta} f(t) & =J^{\alpha+\beta} f(t), \\
J^{\alpha} t^{\gamma} & =\frac{\Gamma(\gamma+1)}{\Gamma(\alpha+\gamma+1)} t^{\alpha+\gamma} .
\end{aligned}
$$

In this study, Caputo definition is used and the fractional derivative of $f(t)$ is defined as

$$
\begin{aligned}
{ }^{C} D_{t}^{\alpha} f(t) & =J^{m-\alpha}\left(\frac{d^{m}}{d t^{m}} f(t)\right) \\
& =\frac{1}{\Gamma(m-\alpha)} \int_{0}^{t} \frac{f^{(m)}(\tau)}{(t-\tau)^{\alpha-m+1}} d \tau,
\end{aligned}
$$

for $m-1<\alpha \leq m, m \in \mathbb{N}, t>0$. The fractional differential operator ${ }^{C} D_{t}^{\alpha}$ is left-inverse (and not right-inverse) to the fractional integral operator $J^{\alpha}$; that is, ${ }^{C} D_{t}^{\alpha} J^{\alpha}=I$, where $I$ is the identity operator. The Laplace transform of the Caputo fractional derivative rule reads

$$
L\left\{{ }^{C} D_{t}^{\alpha} f(t)\right\}=s^{\alpha} F(s)-\sum_{k=0}^{n-1} s^{\alpha-k-1} f^{(k)}(0),
$$

$$
(\alpha>0, n-1<\alpha \leq n) .
$$

Particularly, when $\alpha \in(0,1]$, we have $L\left\{{ }^{C} D_{t}^{\alpha} f(t)\right\}=s^{\alpha} F(s)-$ $s^{\alpha-1} f(0)$. The Laplace transform of the Riemann-Liouville fractional integral rule satisfies

$$
L\left\{J^{\alpha} f(t)\right\}=s^{-\alpha} F(s), \quad(\alpha>0) .
$$

Caputo fractional derivative appears more suitable to be treated by the Laplace transform technique in that it requires the knowledge of the (bounded) initial values of the function and of its integer derivatives of order $k=1,2, \ldots, m-1$, in analogy with the case when $\alpha=n$.

\section{Q-S Synchronization Problem for Integer- Order and Fractional-Order Chaotic System}

We assume that the master chaotic systems can be considered in the following form:

$$
\dot{X}(t)=A X(t)+F(X(t)),
$$

where $X(t) \in \mathbb{R}^{n}$ is the state vector of the master system (6), $A \in \mathbb{R}^{n \times n}$ is a constant matrix, and $F: \mathbb{R}^{n} \rightarrow \mathbb{R}^{n}$ is a nonlinear vector function. Also, consider the slave chaotic system as

$$
{ }^{C} D_{t}^{p} Y(t)=G(Y(t))+U
$$

where $Y(t) \in \mathbb{R}^{m}$ is the state vector of the slave system (7), $G$ : $\mathbb{R}^{m} \rightarrow \mathbb{R}^{m}, p$ is a rational number between 0 and $1,{ }^{C} D_{t}^{p}$ is the Caputo fractional derivative of order $p$, and $U=\left(u_{i}\right)_{1 \leq i \leq m}$ is a vector controller. The problem of $Q-S$ synchronization for the master system (6) and the slave system (7) in dimension $n$ is to find the controller $U$ such that the synchronization error,

$$
e(t)=Q(Y(t))-S(X(t))
$$

satisfies that

$$
\lim _{t \rightarrow+\infty}\|e(t)\|=0
$$

where $Q: \mathbb{R}^{m} \rightarrow \mathbb{R}^{n}, S: \mathbb{R}^{n} \rightarrow \mathbb{R}^{n}$ are continuously differentiable functions. We assume that $n<m$.

\section{General Control Scheme}

The integer-order derivative of the error system (8) can be derived as

$$
\dot{e}(t)=\mathrm{DQ}(Y(t)) \times \dot{Y}(t)-\mathrm{DS}(X(t)) \times \dot{X}(t),
$$

where $\mathbf{D Q}(Y(t)) \in \mathbb{R}^{n \times m}, \mathbf{D} S(X(t)) \in \mathbb{R}^{n \times n}$ are the Jacobian matrices of the functions $Q$ and $S$, respectively,

$$
\mathrm{DQ}(Y(t))=\left(\begin{array}{cccc}
\frac{\partial Q_{1}}{\partial y_{1}} & \frac{\partial Q_{1}}{\partial y_{2}} & \cdots & \frac{\partial Q_{1}}{\partial y_{m}} \\
\frac{\partial Q_{2}}{\partial y_{1}} & \frac{\partial Q_{2}}{\partial y_{2}} & \cdots & \frac{\partial Q_{2}}{\partial y_{m}} \\
\vdots & \vdots & \ddots & \vdots \\
\frac{\partial Q_{n}}{\partial y_{1}} & \frac{\partial Q_{n}}{\partial y_{2}} & \cdots & \frac{\partial Q_{n}}{\partial y_{m}}
\end{array}\right) \text {, }
$$




$$
\operatorname{DS}(X(t))=\left(\begin{array}{cccc}
\frac{\partial S_{1}}{\partial x_{1}} & \frac{\partial S_{1}}{\partial x_{2}} & \cdots & \frac{\partial S_{1}}{\partial x_{n}} \\
\frac{\partial S_{2}}{\partial x_{1}} & \frac{\partial S_{2}}{\partial x_{2}} & \cdots & \frac{\partial S_{2}}{\partial x_{n}} \\
\vdots & \vdots & \ddots & \vdots \\
\frac{\partial S_{n}}{\partial x_{1}} & \frac{\partial S_{n}}{\partial x_{2}} & \cdots & \frac{\partial S_{n}}{\partial x_{n}}
\end{array}\right)
$$

Hence, we have the following result.

Theorem 1. There exists a suitable feedback gain matrix $C \in$ $\mathbb{R}^{n \times n}$ to realize the $Q-S$ synchronization between the master system (6) and the slave system (7) in n-D under the following controller:

$$
U=-G(Y(t))+J^{1-p}(G(Y(t))+V),
$$

where the vector quantity $V=\left(V_{i}\right)_{1 \leq i \leq m}$ is defined as

$$
\begin{aligned}
\left(V_{1}, V_{2}, \ldots, V_{n}\right)^{T} & =-M^{-1} R, \\
\left(V_{n+1}, V_{n+2}, \ldots, V_{m}\right)^{T} & =0,
\end{aligned}
$$

where $M^{-1}$ is the inverse of matrix $M$,

$$
\begin{aligned}
M= & \left(\begin{array}{cccc}
\frac{\partial Q_{1}}{\partial y_{1}} & \frac{\partial Q_{1}}{\partial y_{2}} & \cdots & \frac{\partial Q_{1}}{\partial y_{n}} \\
\frac{\partial Q_{2}}{\partial y_{1}} & \frac{\partial Q_{2}}{\partial y_{2}} & \cdots & \frac{\partial Q_{2}}{\partial y_{n}} \\
\vdots & \vdots & \ddots & \vdots \\
\frac{\partial Q_{n}}{\partial y_{1}} & \frac{\partial Q_{n}}{\partial y_{2}} & \cdots & \frac{\partial Q_{n}}{\partial y_{n}}
\end{array}\right), \\
R= & (C-A) e(t)+\mathrm{DQ}(Y(t)) \times G(Y(t)) \\
& -\mathrm{DS}(X(t)) \times(A X(t)+F(X(t))) .
\end{aligned}
$$

Proof. By inserting the control law described by (12) into (7), we can rewrite the slave system as follows:

$$
{ }^{C} D_{t}^{p} Y(t)=J^{1-p}(G(Y(t))+V) .
$$

Applying the Laplace transform to (17) and letting $\mathbf{F}(s)=$ $\mathbf{L}(Y(t))$, we obtain

$$
s^{p} \mathbf{F}(s)-s^{p-1} Y(0)=s^{p-1} \mathbf{L}(G(Y(t))+V) ;
$$

multiplying both the left-hand and right-hand sides of (18) by $s^{1-p}$ and applying the inverse Laplace transform to the result, we obtain a new equation for the slave system

$$
\dot{Y}(t)=G(Y(t))+V .
$$

Now, the error system (10) can be described as

$$
\begin{aligned}
\dot{e}(t)= & (A-C) e(t)+\mathrm{DQ}(Y(t)) \times G(Y(t)) \\
& +(C-A)(Q(Y(t))-S(X(t)))-\mathrm{D} S(X(t)) \\
& \times(A X(t)+F(X(t)))+\mathrm{DQ}(Y(t)) \times V,
\end{aligned}
$$

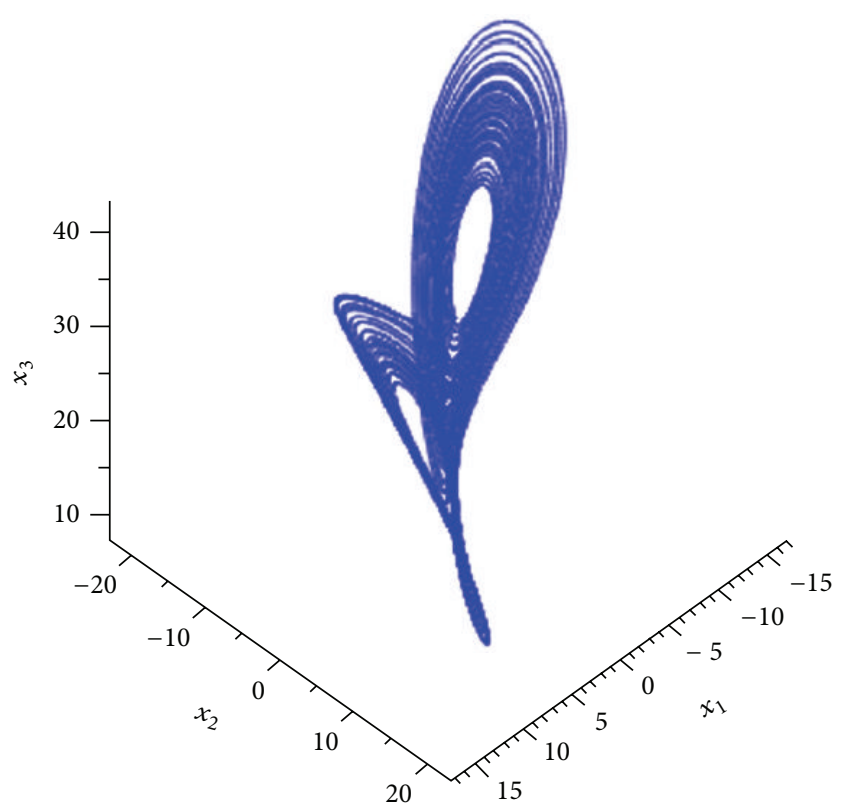

FIgURE 1: The chaotic attractor of Lorenz system when $(\alpha, \beta, \gamma)=$ $(10,28,-8 / 3)$.

where $C \in \mathbb{R}^{n \times n}$ is a feedback gain matrix to be chosen. The error system (20) can be simplified as follows:

$$
\dot{e}(t)=(A-C) e(t)+R+\mathrm{DQ}(Y(t)) \times V,
$$

where $R$ was defined by (16). By using (14), the error system (21) can be written as

$$
\dot{e}(t)=(A-C) e(t)+R+M \times\left(V_{1}, V_{2}, \ldots, V_{n}\right)^{T} .
$$

Hence, by substituting (13) into (22), we get

$$
\dot{e}(t)=(A-C) e(t) \text {. }
$$

With respect to the asymptotic stability property of linear continuous-time dynamical systems, if the feedback gain matrix $C$ is selected such that all eigenvalues of $A-C$ are strictly negative, it is immediate that all solutions of the error system (23) go to zero as $t \rightarrow \infty$. Therefore, systems (6) and (7) are globally Q-S synchronized in $n$-D.

\section{Illustrative Example}

In this section, to validate the Q-S synchronization method proposed in the previous section, we consider the Lorenz system as a master system and the fractional-order hyperchaotic Lorenz system as a slave system. The Lorenz system can be described as

$$
\begin{aligned}
& \dot{x}_{1}=\alpha\left(x_{2}-x_{1}\right), \\
& \dot{x}_{2}=\beta x_{1}-x_{2}-x_{1} x_{3}, \\
& \dot{x}_{3}=\gamma x_{3}+x_{1} x_{2},
\end{aligned}
$$




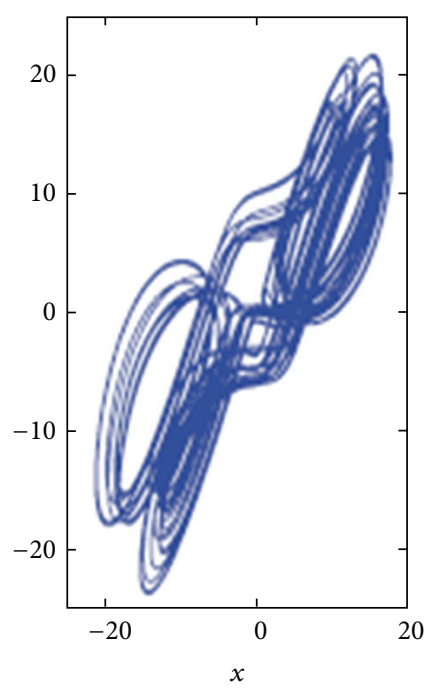

(a)

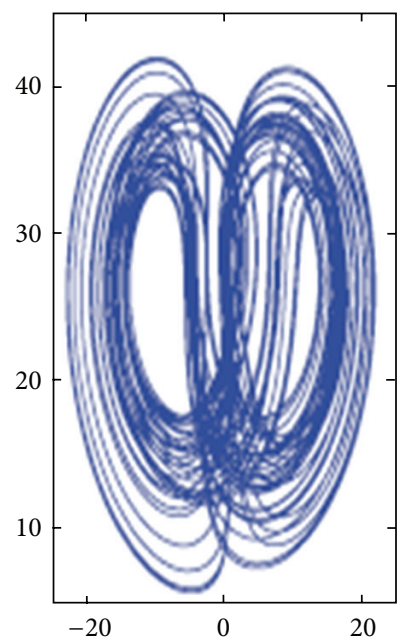

(d)

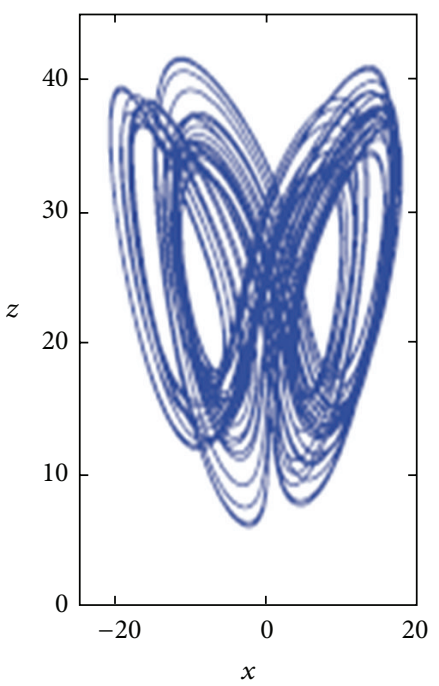

(b)

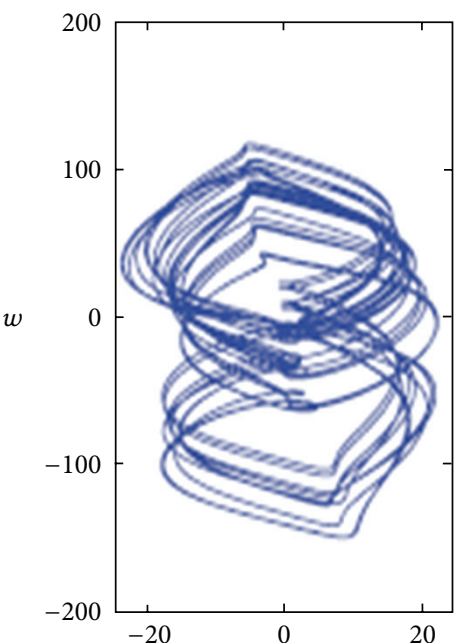

(e)

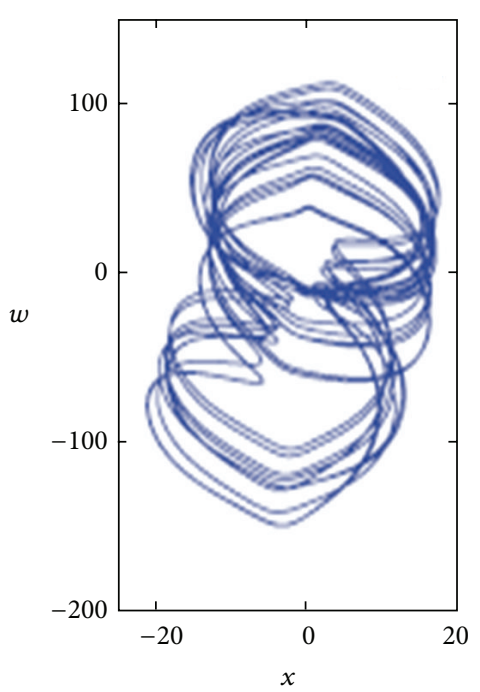

(c)

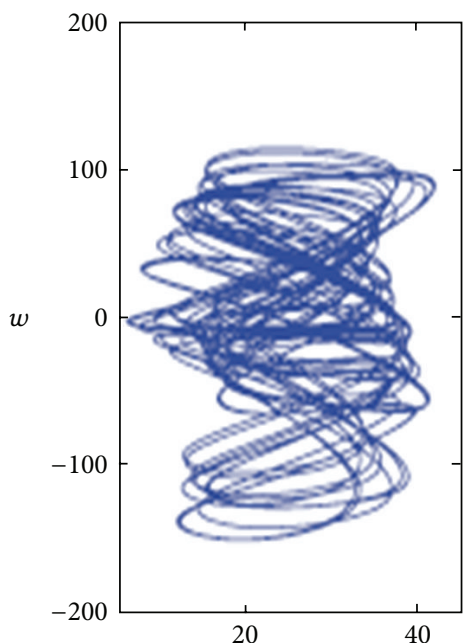

(f)

FIGURE 2: Chaotic attractor projections of fractional-order hyperchaotic Lorenz system when $p=0.98$ and $(a, b, c, r)=(10,8 / 3,28,-1)$.

which has a chaotic attractor, for example, when $(\alpha, \beta, \gamma)=$ $(10,28,-8 / 3)[45]$. Then the linear part $A=\left(a_{i j}\right)_{3 \times 3}$ and the nonlinear part $F$ of the Lorenz system (24) are given by

$$
\begin{aligned}
& A=\left(\begin{array}{ccc}
-\alpha & \alpha & 0 \\
\beta & -1 & 0 \\
0 & 0 & \gamma
\end{array}\right), \\
& F=\left(\begin{array}{c}
0 \\
-x_{1} x_{3} \\
x_{1} x_{2}
\end{array}\right) .
\end{aligned}
$$

The Lorenz chaotic attractor is shown in Figure 1.

The controlled fractional-order hyperchaotic Lorenz system can be described by the following nonlinear fractionalorder ODE:

$$
\begin{aligned}
& D^{p} y_{1}=a\left(y_{2}-y_{1}\right)+y_{4}+u_{1}, \\
& D^{p} y_{2}=c y_{1}-y_{2}-y_{1} y_{3}+u_{2},
\end{aligned}
$$

$$
\begin{aligned}
& D^{p} y_{3}=-b y_{3}+y_{1} y_{2}+u_{3}, \\
& D^{p} y_{4}=r y_{4}-y_{2} y_{3}+u_{4},
\end{aligned}
$$

where $u_{i}, i=1,2,3,4$, are the controllers. This system, as shown in [46], exhibits hyperchaotic behaviors when $p=0.98$ and $(a, b, c, r)=(10,8 / 3,28,-1)$. Chaotic attractor projections of the fractional-order hyperchaotic Lorenz system are shown in Figure 2.

According to Q-S synchronization control technique proposed in the previous section, the functions $Q: \mathbb{R}^{4} \rightarrow \mathbb{R}^{3}$ and $S: \mathbb{R}^{3} \rightarrow \mathbb{R}^{3}$ are selected as follows:

$$
\begin{aligned}
& Q\left(y_{1}, y_{2}, y_{3}, y_{4}\right) \\
& =\left(y_{1}+\frac{1}{3} y_{1}^{3}+y_{4}, y_{2}+2 y_{4}, y_{3}+3 y_{4}\right)^{T}, \\
& S\left(x_{1}, x_{2}, x_{3}\right)=\left(x_{1}, x_{2}, x_{3}+x_{1}\right)^{T} .
\end{aligned}
$$


Then,

$$
\begin{aligned}
\mathbf{D} Q\left(y_{1}, y_{2}, y_{3}, y_{4}\right) & =\left(\begin{array}{ccccc}
1+y_{1}^{2} & 0 & 0 & 1 \\
0 & 1 & 0 & 2 \\
0 & 0 & 1 & 3
\end{array}\right), \\
\operatorname{DS}\left(x_{1}, x_{2}, x_{3}\right) & =\left(\begin{array}{lll}
1 & 0 & 0 \\
0 & 1 & 0 \\
1 & 0 & 1
\end{array}\right) .
\end{aligned}
$$

It is easy to show that if we choose the feedback gain matrix $C$ as

$$
C=\left(\begin{array}{ccc}
0 & 10 & 0 \\
28 & 0 & 0 \\
0 & 0 & 0
\end{array}\right),
$$

then the eigenvalues of the matrix $A-C$ are strictly negative. Then the controllers $u_{1}, u_{2}, u_{3}$, and $u_{4}$ can be designed as follows:

$$
\begin{aligned}
u_{1} & =J^{0.02}\left[-\alpha e_{1}(t)\right. \\
& \left.-\frac{1}{1+y_{1}^{2}}\left(r y_{4}-y_{2} y_{3}-\alpha\left(x_{2}-x_{1}\right)\right)\right]-a\left(y_{2}\right. \\
& \left.-y_{1}\right)-y_{4}, \\
u_{2} & =-c y_{1}+y_{2}+y_{1} y_{3}+J^{0.02}\left[-e_{2}(t)-2 r y_{4}+2 y_{2} y_{3}\right. \\
& \left.+\beta x_{1}-x_{2}-x_{1} x_{3}\right], \\
u_{3} & =b y_{3}-y_{1} y_{2}+J^{0.02}\left[-\gamma e_{3}(t)-3 r y_{4}+3 y_{2} y_{3}\right. \\
& \left.+\alpha\left(x_{2}-x_{1}\right)+\gamma x_{3}+x_{1} x_{2}\right], \\
u_{4} & =-r y_{4}+y_{2} y_{3}+J^{0.02}\left[r y_{4}-y_{2} y_{3}\right],
\end{aligned}
$$

and the error system can be written as

$$
\begin{aligned}
& \dot{e}_{1}=-10 e_{1}, \\
& \dot{e}_{2}=-e_{2}, \\
& \dot{e}_{3}=-\frac{8}{3} e_{3} .
\end{aligned}
$$

Therefore, according to Theorem 1, systems (24) and (26) are globally $Q-S$ synchronized in 3D. The numerical simulations of the error functions evolution are shown in Figure 3.

\section{Conclusion}

In this paper, new control scheme, based on fractional control law, Laplace transformation, and stability theory of linear integer-order dynamical system, for $Q-S$ synchronization was presented between $n$-dimensional integer-order master

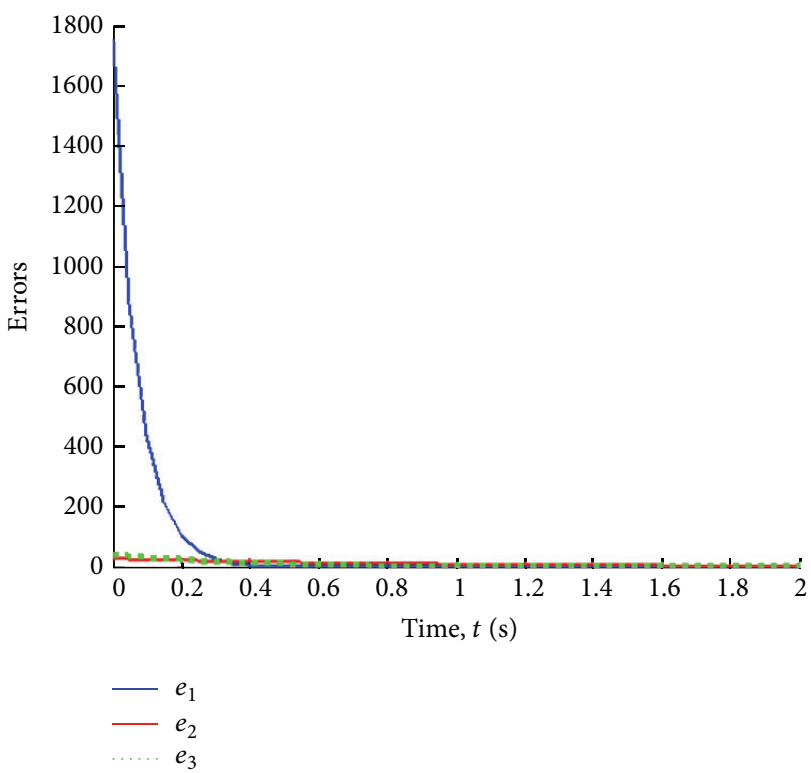

FIgURE 3: Time evolution of Q-S synchronization errors between systems (24) and (26).

system and $m$-dimensional fractional-order slave system. To observe $Q-S$ synchronization with respect to dimension $n$, the synchronization criterion was obtained via controlling the linear part of the master system. Numerical example and simulations result were used to verify the effectiveness of the proposed control method.

\section{Conflict of Interests}

The authors declare that there is no conflict of interests regarding the publication of this paper.

\section{References}

[1] I. Petráš, "A note on the fractional-order Chua’s system," Chaos, Solitons and Fractals, vol. 38, no. 1, pp. 140-147, 2008.

[2] D. Cafagna, "Fractional calculus: a mathematical tool from the past for present engineers," IEEE Industrial Electronics Magazine, vol. 1, no. 2, pp. 35-40, 2007.

[3] P. J. Torvik and R. L. Bagley, "On the appearance of the fractional derivative in the behavior of real materials," Journal of Applied Mechanics, vol. 51, no. 2, pp. 294-298, 1984.

[4] B. J. West, M. Bologna, and P. Grigolini, Physics of Fractal Operators, Springer, New York, NY, USA, 2002.

[5] S. Westerlund, Dead Matter Has Memory!, Causal Consulting, Kalmar, Sweden, 2002.

[6] A. Oustaloup, La Derivation Non Entiere: Theorie, Synthese et Applications, Hermès, Paris, France, 1995.

[7] G.-Q. Si, Z.-Y. Sun, and Y.-B. Zhang, "A general method for synchronizing an integer-order chaotic system and a fractionalorder chaotic system," Chinese Physics B, vol. 20, no. 8, Article ID 080505, 2011.

[8] Z. Ping, Y.-M. Cheng, and K. Fei, "Synchronization between fractional-order chaotic systems and integer orders chaotic 
systems (fractional-order chaotic systems)," Chinese Physics $B$, vol. 19, no. 9, Article ID 090503, 2010.

[9] D.-Y. Chen, R.-F. Zhang, X.-Y. Ma, and J. Wang, "Synchronization between a novel class of fractional-order and integer-order chaotic systems via a sliding mode controller," Chinese Physics B, vol. 21, no. 12, Article ID 120507, 2012.

[10] D. Chen, R. Zhang, J. C. Sprott, H. Chen, and X. Ma, "Synchronization between integer-order chaotic systems and a class of fractional-order chaotic systems via sliding mode control," Chaos, vol. 22, Article ID 023130, 2012.

[11] Y.-P. Wu and G.-D. Wang, "Synchronization between fractionalorder and integer-order hyperchaotic systems via sliding mode controller," Journal of Applied Mathematics, vol. 2013, Article ID 151025, 5 pages, 2013.

[12] Y. Wu and G. Wang, "Synchronization of a class of fractionalorder and integer order hyperchaotic systems," Journal of Vibration and Control, vol. 20, no. 10, pp. 1584-1588, 2013.

[13] L.-X. Jia, H. Dai, and M. Hui, "Nonlinear feedback synchronisation control between fractional-order and integer-order chaotic systems," Chinese Physics B, vol. 19, no. 11, Article ID 110509, 2010.

[14] I. El Gammoudi and M. Feki, "Synchronization of integer order and fractional order Chua's systems using robust observer," Communications in Nonlinear Science and Numerical Simulation, vol. 18, no. 3, pp. 625-638, 2013.

[15] D. Chen, C. Wu, H. H. Iu, and X. Ma, "Circuit simulation for synchronization of a fractional-order and integer-order chaotic system," Nonlinear Dynamics, vol. 73, no. 3, pp. 1671-1686, 2013.

[16] A. Khan and P. Tripathi, "Synchronization between a fractional order chaotic system and an integer order chaotic system," Nonlinear Dynamics and Systems Theory, vol. 14, no. 4, 2013.

[17] L.-X. Yang, W.-S. He, and X.-J. Liu, "Synchronization between a fractional-order system and an integer order system," Computers \& Mathematics with Applications, vol. 62, no. 12, pp. 47084716, 2011.

[18] Y. Wu and G. Wang, "Synchronization and anti-synchronization between a class of fractional-order and integer-order chaotic systems with only one controller term," Journal of Theoretical \& Applied Information Technology, vol. 48, no. 1, pp. 145-151, 2013.

[19] Z. Ping and Y.-X. Cao, "Function projective synchronization between fractional-order chaotic systems and integer-order chaotic systems," Chinese Physics B, vol. 19, no. 10, Article ID 100507, 2010.

[20] H.-J. Liu, Z.-L. Zhu, H. Yu, and Q. Zhu, "Modified function projective synchronization of fractional order chaotic systems with different dimensions," Discrete Dynamics in Nature and Society, vol. 2013, Article ID 763564, 7 pages, 2013.

[21] Z. Wu, X. Xu, G. Chen, and X. Fu, "Generalized matrix projective synchronization of general colored networks with different-dimensional node dynamics," Journal of the Franklin Institute, vol. 351, no. 9, pp. 4584-4591, 2014.

[22] G. Zhang, Z. Liu, and Z. Ma, "Generalized synchronization of different dimensional chaotic dynamical systems," Chaos, Solitons \& Fractals, vol. 32, no. 2, pp. 773-779, 2007.

[23] Y. Yu, H.-X. Li, and J. Yu, "Generalized synchronization of different dimensional chaotic systems based on parameter identification," Modern Physics Letters B, vol. 23, no. 22, pp. 2593-2606, 2009.

[24] X. He, C. Li, J. Huang, and L. Xiao, "Generalized synchronization of arbitrary-dimensional chaotic systems," Optik, vol. 126, pp. 454-461, 2015.
[25] A. Ouannas and Z. Odibat, "Generalized synchronization of different dimensional chaotic dynamical systems in discrete time," Nonlinear Dynamics, vol. 81, no. 1-2, pp. 765-771, 2015.

[26] M. Hu, Z. Xu, R. Zhang, and A. Hu, "Adaptive full state hybrid projective synchronization of chaotic systems with the same and different order," Physics Letters A, vol. 365, no. 4, pp. 315-327, 2007.

[27] A. M. El-Sayed, H. M. Nour, A. Elsaid, A. E. Matouk, and A. Elsonbaty, "Circuit realization, bifurcations, chaos and hyperchaos in a new 4D system," Applied Mathematics and Computation, vol. 239, pp. 333-345, 2014.

[28] S. Ogunjo, "Increased and reduced order synchronization of 2D and 3D dynamical systems," International Journal of Nonlinear Science, vol. 16, no. 2, pp. 105-112, 2013.

[29] M. M. Al-Sawalha and M. S. M. Noorani, "Adaptive increasingorder synchronization and anti-synchronization of chaotic systems with uncertain parameters," Chinese Physics Letters, vol. 28, no. 11, Article ID 110507, 2011.

[30] K. S. Ojo, S. T. Ogunjo, A. N. Njah, and I. A. Fuwape, "Increasedorder generalized synchronization of chaotic and hyperchaotic systems," Pramana, vol. 84, no. 1, pp. 33-45, 2015.

[31] H. Manfeng and X. Zhenyuan, "A general scheme for Q-S synchronization of chaotic systems," Nonlinear Analysis. Theory, Methods \& Applications, vol. 69, no. 4, pp. 1091-1099, 2008.

[32] Q. Wang and Y. Chen, "Generalized Q-S (lag, anticipated and complete) synchronization in modified Chua's circuit and Hindmarsh-rose systems," Applied Mathematics and Computation, vol. 181, no. 1, pp. 48-56, 2006.

[33] Z. L. Wang and X.-R. Shi, "Adaptive Q-S synchronization of non-identical chaotic systems with unknowns parameters," Nonlinear Dynamics, vol. 59, no. 4, pp. 559-567, 2010.

[34] Z. Yan, "Q-S (lag or anticipated) synchronization backstepping scheme in a class of continuous-time hyperchaotic systems-a symbolic-numeric computation approach," Chaos, vol. 15, no. 2, Article ID 023902, 2005.

[35] Y. Yang and Y. Chen, “The generalized Q-S synchronization between the generalized Lorenz canonical form and the Rössler system," Chaos, Solitons and Fractals, vol. 39, no. 5, pp. 23782385, 2009.

[36] J. Zhao and T. Ren, "Q-S synchronization between chaotic systems with double scaling functions," Nonlinear Dynamics, vol. 62, no. 3, pp. 665-672, 2010.

[37] J. Zhao and K. Zhang, "A general scheme for Q-S synchronization of chaotic systems with unknown parameters and scaling functions," Applied Mathematics and Computation, vol. 216, no. 7, pp. 2050-2057, 2010.

[38] Z. Yan, "Q-S synchronization in 3D Hénon-like map and generalized Hénon map via a scalar controller," Physics Letters A, vol. 342, no. 4, pp. 309-317, 2005.

[39] L.-X. Yang and W.-S. He, "Adaptive Q-S synchronization of fractional-order chaotic systems with nonidentical structures," Abstract and Applied Analysis, vol. 2013, Article ID 367506, 8 pages, 2013.

[40] K. B. Oldham and J. Spanier, The Fractional Calculus, Academic Press, New York, NY, USA, 1974.

[41] K. S. Miller and B. Ross, An Introduction to the Fractional Calculus and Fractional Differential Equations, Wiley, New York, NY, USA, 1993.

[42] M. Caputo, "Linear models of dissipation whose Q is almost frequency independent. II," Geophysical Journal International, vol. 13 , no. 5 , pp. 529-539, 1967. 
[43] I. Podlubny, Fractional Differential Equations, Academic Press, New York, NY, USA, 1999.

[44] R. Goren and F. Mainardi, "Fractional calculus: integral and differential equations of fractional order," in Fractals and Fractional Calculus in Continuum Mechanics, A. Carpinteri and F. Mainardi, Eds., Springer, New York, NY, USA, 1997.

[45] E. N. Lorenz, "Deterministic nonperiodic flow," Journal of the Atmospheric Sciences, vol. 20, no. 2, pp. 130-141, 1963.

[46] Z. Wang, X. Huang, Y.-X. Li, and X.-N. Song, "A new image encryption algorithm based on the fractional-order hyperchaotic Lorenz system," Chinese Physics B, vol. 22, no. 1, Article ID 010504, 2013. 

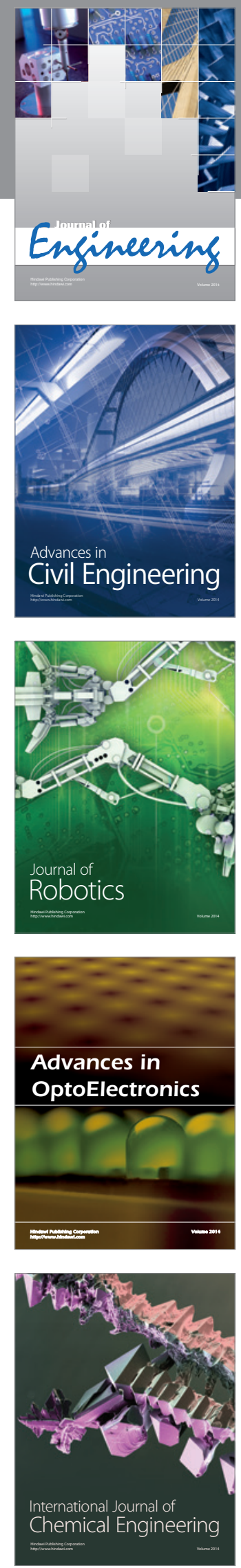

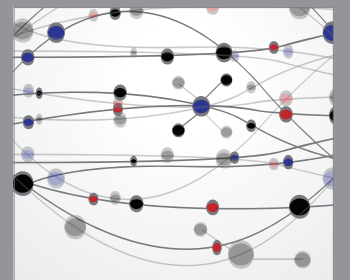

The Scientific World Journal
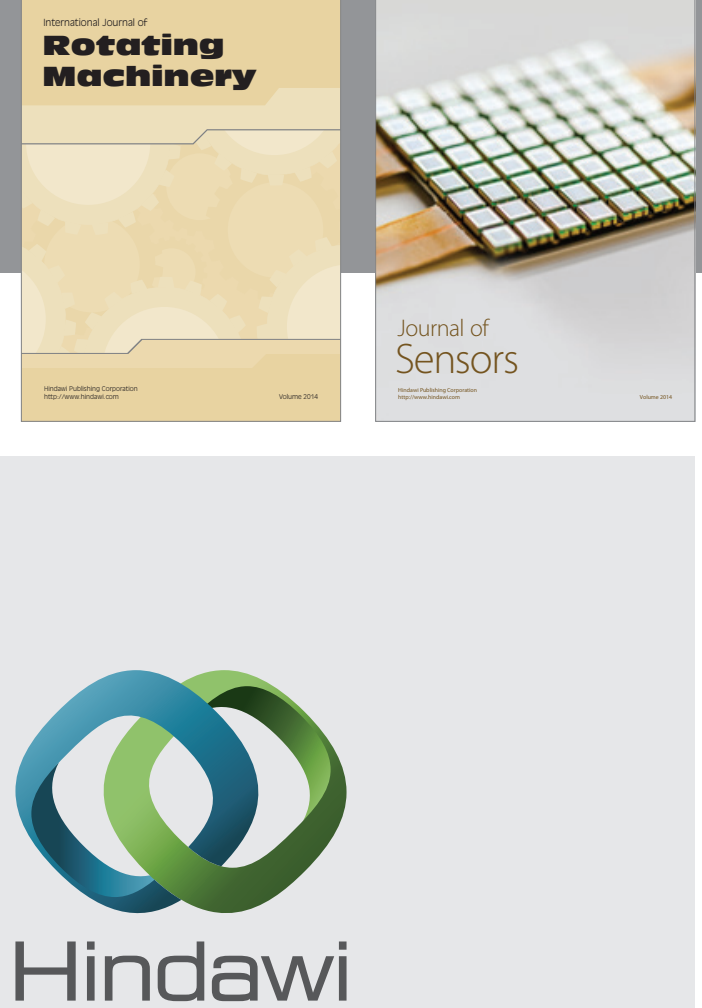

Submit your manuscripts at http://www.hindawi.com
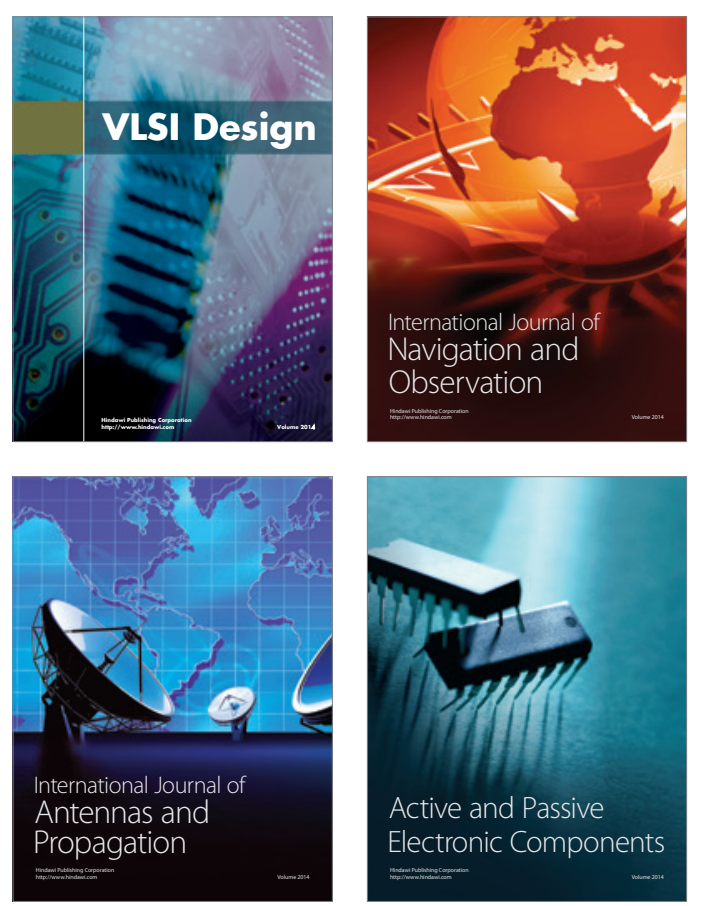
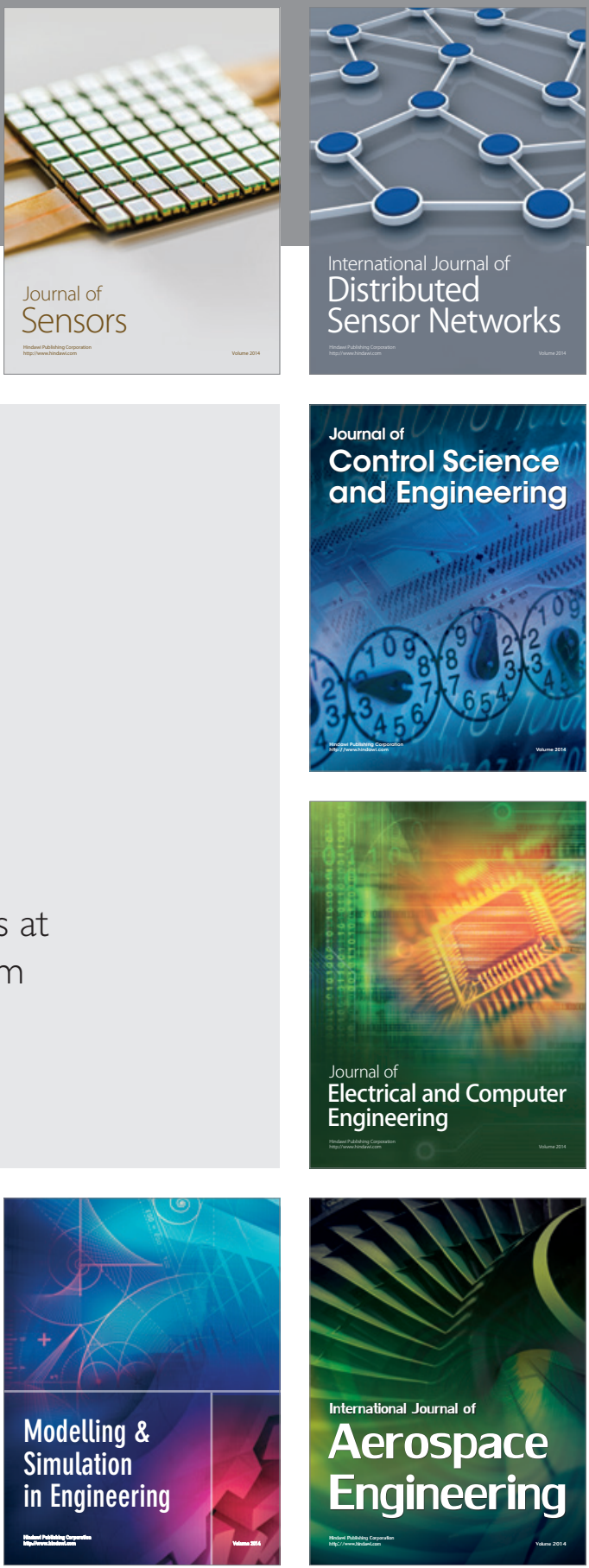

Journal of

Control Science

and Engineering
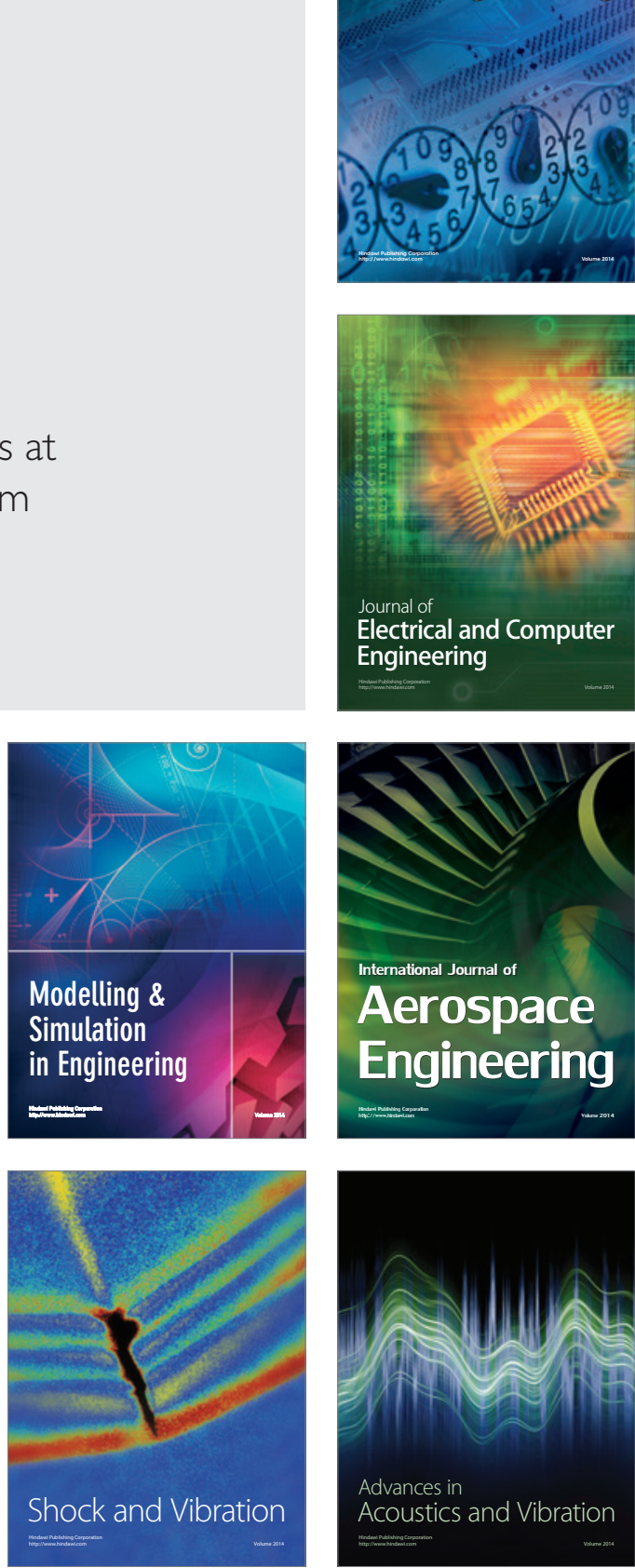